\title{
Determinantes de violencia de pareja en trabajadoras del IMSS Morelos
}

\author{
Paola Adanari Ortega-Ceballos, MC, ${ }^{(1)}$ Jyoti Mudgal, PhD, (2) Yvonne Flores, PhD, ${ }^{(2)}$ \\ Leonor Rivera-Rivera, MC, ${ }^{(3)}$ Juan Carlos Díaz-Montiel, M en C, ${ }^{(2)}$ Jorge Salmerón, D en C. ${ }^{(2)}$
}

\author{
Ortega-Ceballos PA, Mudgal J, Flores Y \\ Rivera-Rivera L, Díaz-Montiel JC, Salmerón J. \\ Determinantes de violencia de pareja \\ en trabajadoras del IMSS, Morelos. \\ Salud Publica Mex 2007;49:357-366.
}

\section{Resumen}

Objetivo. Identificar la ocurrencia de violencia de pareja y evaluar los factores asociados a este fenómeno en una muestra de trabajadoras del Instituto Mexicano del Seguro Social (IMSS) Morelos. Material y métodos. Un total de I I73 mujeres de la cohorte de trabajadores del IMSS proporcionó información sobre frecuencia y percepción de severidad de violencia psicológica, física o sexual durante los últimos 12 meses. El estudio se realizó en el estado de Morelos, entre octubre de 1998 y marzo de 2000 . Se emplearon modelos de regresión logística politómica a fin de estimar razones de momios para distintos grados de violencia. Resultados. Se observó una alta prevalencia de violencia. Los principales factores asociados fueron la relación de pareja y el consumo de alcohol; el estado emocional de la pareja en el hogar; la tensión laboral de la mujer y los antecedentes de violencia en la infancia. Conclusiones. Los principales factores determinantes de violencia de pareja son potencialmente modificables a través de intervenciones que ayuden a manejar el nivel de tensión. Es necesario tomar en cuenta estos hallazgos al planear programas preventivos de violencia de pareja en México.

Palabras clave: ocurrencia; severidad; violencia doméstica; México
Ortega-Ceballos PA, Mudgal J, Flores Y,

Rivera-Rivera L, Díaz-Montiel JC, Salmerón J.

Determinants of partner violence

in health workers of IMSS, Morelos.

Salud Publica Mex 2007;49:357-366.

\section{Abstract}

Objective. To study the prevalence of partner violence, and to identify the associated risk factors in a sample of female workers of IMSS (Mexican Social Security Institute), Morelos State. Material and Methods. Cross-sectional data from I 173 women participating in the cohort study of IMSS workers are utilized to study these associations. The study provides information on frequency of psychological, physical or sexual violence and perception of severity during the 12 months prior to the time of data collection. It was carried out in Morelos between October 1998 and March 2000. Polytomous logistic regression models were used to obtain odds ratios for different degrees of partner violence. Results. A high prevalence of partner violence is observed in the sample. Main factors associated with higher severity of violence are state of the relationship and alcohol intake, emotional status of the couple at home, work burden of the woman, and a history of violence in childhood. Conclusions. All these factors are potentially modifiable through interventions aimed at stress reduction. These results should be considered when developing preventive programs against partner violence in Mexico.

Key words: occurrence; severity; domestic violence; Mexico

(I) Secretaría Académica. Instituto Nacional de Salud Pública. Cuernavaca, México.

(2) Hospital General No. I. Instituto Mexicano del Seguro Social. Cuernavaca, México.

(3) Centro de Investigaciones en Salud Poblacional. Instituto Nacional de Salud Pública. Cuernavaca, México.

Fecha de recibido: 23 de enero de 2006 - Fecha de aceptado: 21 de marzo de 2007

Solicitud de sobretiros: M en C Paola Adanari Ortega-Ceballos. Secretaría Académica. Instituto Nacional de Salud Pública. Avenida Universidad 655, Col. Santa María Ahuacatitlán, 62508 Cuernavaca, Morelos, México. Correo electrónico: portega79@yahoo.com.mx 
L a Organización Mundial de la Salud define la violencia de pareja como "la gama de actos sexuales, psicológicos y físicos coercitivos, usados sobre las mujeres por su pareja". ${ }^{1}$ En la sociedad mexicana existen relaciones estructurales de desigualdad de género donde los niños y las niñas aprenden que los hombres dominan y que la violencia es un medio aceptable para reafirmar su poder, subordinar a las mujeres y establecer el orden. ${ }^{2-4}$

La violencia de pareja constituye un problema de salud pública que amerita políticas públicas con obligaciones específicas para distintas instituciones, incluidas las de salud. ${ }^{5}$ En las últimas décadas los problemas de salud derivados de la violencia de pareja han ocupado la atención en distintos estudios. ${ }^{6,7}$ Se ha observado que las mujeres maltratadas tienen complicaciones durante el parto y posparto y corren mayor riesgo de tener recién nacidos de bajo peso que las mujeres no maltratadas. ${ }^{8}$ Las mujeres mueren a causa de agresiones por parte de sus parejas en una proporción ocho veces superior a la de los hombres. ${ }^{9}$

En diferentes países se han documentado altas prevalencias de violencia de pareja; así, en Europa durante el último año fueron de 6.2, 3.9 y 1.2\% de violencia psicológica, física y sexual, respectivamente. ${ }^{10}$ En Latinoamérica se documentaron prevalencias de violencia física durante el último año entre 3 y $27 \%,{ }^{11}$ mientras que para la violencia sexual las prevalencias fluctúan entre $4^{12}$ y 47 por ciento. ${ }^{11}$ En México, y de acuerdo con los resultados de algunas encuestas nacionales, de 19.6 a $36.6 \%$ de las mujeres son víctimas de violencia psicológica y de 9.3 a 10.3\% sufren violencia física. La violencia sexual es la menos frecuente (7.0-7.8\%). Al agrupar los diversos tipos de violencia, las encuestas nacionales indican que de 21 a $44 \%$ de las mujeres mexicanas experimentan algún tipo de violencia de pareja. ${ }^{13-15}$

Entre los factores que se han asociado con la violencia de pareja se encuentran la baja edad de las mujeres, ${ }^{16}$ un nivel socioeconómico bajo, ${ }^{5,17}$ el presenciar o sufrir violencia intrafamiliar durante la niñez, ${ }^{5,13,17}$ un nivel bajo de escolaridad, ${ }^{16,17}$ el estado civil, ${ }^{17}$ y el consumo excesivo de alcohol o drogas por parte de algún miembro de la familia. ${ }^{18-21}$

Gran parte de los estudios sobre violencia de pareja se han realizado con la participación de usuarias de servicios de salud, ${ }^{13}$ son pocas las investigaciones que se han llevado a cabo con prestadores de servicios de salud. ${ }^{22}$ En la mayoría de estos estudios se ha buscado conocer la percepción de la violencia y no los determinantes de victimización. Los esfuerzos por lograr que se enfrente activamente el problema de violencia de pareja, se ven obstaculizados por la falta de datos sobre sus determinantes. En un intento por responder a esta necesidad de información, el presente estudio identifica la ocurrencia y severidad de distintos tipos de violencia de pareja y evalúa los factores asociados con este fenómeno en una muestra de mujeres prestadoras de servicios de salud del Instituto Mexicano del Seguro Social (IMSS) Morelos.

\section{Material y métodos}

El proyecto de investigación "Cohorte de trabajadores IMSS" es un estudio diseñado para evaluar la asociación entre factores de estilo de vida y la ocurrencia de enfermedades crónicas. La investigación fue aprobada por el Comité Local de Investigación del Hospital General Regional del IMSS Morelos.

La población de estudio está conformada por mujeres trabajadoras, activas o jubiladas, de los servicios médicos del IMSS Morelos. La invitación a participar en el mismo estuvo precedida por una labor de sensibilización para exponer los objetivos del proyecto, que se llevó a cabo en el área de trabajo de las mujeres, y de manera personal. Se invitó a 2980 mujeres a participar en la medición basal de la Cohorte del IMSS (1998-2000), de las cuales se logró reclutar exitosamente a 2559 (tasa de respuesta de 86\%). La recolección de información se realizó en dos tiempos, por medio de cuestionarios autoaplicables. Se incluyó a las mujeres que contaban con pareja al momento del levantamiento de la información y que tenían información completa sobre violencia, quedando así un total 1173 mujeres para análisis. No se observaron diferencias importantes entre distintas características sociodemográficas (edad, escolaridad, categoría laboral) entre la población analizada y la potencialmente elegible, ni entre las mujeres que respondieron y aquellas que no lo hicieron.

Por medio del cuestionario autoaplicable, las participantes proporcionaron datos, tanto sobre sí mismas como sobre su pareja, vinculados con una serie de factores sociodemográficos y de estilos de vida. Por medio de estos cuestionarios, se colectó información detallada sobre los episodios de violencia psicológica, física o sexual perpetrada por su pareja durante el último año. Este instrumento se ha utilizado en estudios realizados anteriormente por los autores del presente artículo, y ha quedado demostrada su eficacia para acopiar información sobre violencia de pareja en la sociedad mexicana. ${ }^{17}$ Así, a través del mismo se obtienen datos para la conceptualización de la violencia de pareja como un patrón caracterizando por: a) violencia física, conformada por una serie de conductas de agresión física de las que son víctimas las mujeres (anexo 1: 1-15, 20, $21)$; b) violencia psicológica, integrada por una serie de conductas emocionalmente dominadoras y represivas 
de la pareja hacia la mujer (anexo 1: 16-19, 22-30, 32); c) violencia sexual, que implica forzar físicamente a la mujer a tener la relación sexual (anexo 1: 31, 33, 34, 35). La serie de reactivos que componen los diferentes tipos de violencia se encuentran enlistados en el anexo 1.

Por otra parte, también se obtuvo información acerca de la percepción de la mujer sobre la severidad de distintos actos de violencia; aquí las participantes otorgaron un valor a cada acto de violencia, de acuerdo con su percepción, en un rango de 1 a 100 (anexo 1).

\section{Medieión de la violencia}

\section{Indicador de frecuencia relativa de violencia}

A partir de la información sobre la existencia de los distintos actos de violencia de pareja durante el último año, así como la frecuencia de cada uno de ellos (17 actos de violencia física, 14 de violencia psicológica y cuatro de violencia sexual), se generó un "indicador de frecuencia relativa" para cada tipo de violencia. Este indicador permitió conjuntar el simple antecedente de violencia de pareja (sí/no) con la frecuencia con que dicho evento se presentaba, lo cual brinda una mejor estimación de la frecuencia relativa de este fenómeno. En la creación de ese indicador, cada acto de violencia de pareja fue ponderado por la frecuencia relativa correspondiente ( $1=$ una vez; $3=$ varias veces; $9=$ muchas veces). A continuación, esos valores ponderados fueron sumados por tipo específico de violencia de pareja y, posteriormente, se clasificaron de la siguiente manera: a) para la violencia psicológica y física; no violencia, baja-moderada y alta. El nivel alto de violencia corresponde al tercil superior; b) para la violencia sexual; no violencia, baja y alta. El nivel alto corresponde al segundo y tercer tercil.

\section{Indicador de severidad de violencia}

En forma paralela se construyó un "indicador de severidad de violencia de pareja", según los procedimientos sugeridos por Castro R. y colaboradores. ${ }^{5}$ De acuerdo con el valor asignado por las mujeres a cada acto de violencia (rango 1 a 100), se obtuvo el promedio de cada uno de ellos; posteriormente, se sumaron los promedios de todos los actos que conformaban cada tipo de violencia. Los valores promedios fueron de 0.77 para violencia psicológica, 0.83 para violencia física y 0.88 para violencia sexual. Para obtener el indicador de severidad de violencia, se multiplicó la frecuencia con que ocurrió cada acto de violencia de pareja (1=una vez, $2=$ varias veces, $3=$ muchas veces) por el valor ponderado de la percepción para cada tipo de violencia (valores de .049 a 4.03). Para fines de análisis, se establecieron tres categorías: mujeres que indicaron no haber sido víctimas de violencia; mujeres en el nivel de severidad de violencia baja-moderada, y mujeres con nivel alto de severidad de violencia de pareja.

\section{Medición de variables de exposición}

Se construyó un indicador de relación de pareja y consumo de alcohol, a partir de las siguientes cuatro variables que respondieron las participantes en el estudio: a) ¿Su pareja actual tiene otra mujer?: sí, no, no sé. b) La última vez que tomó su esposo o pareja algún tipo de bebida alcohólica, ¿cuántas copas tomó?: nunca ha tomado; una copa; dos copas; tres copas; cuatro copas; cinco copas; seis copas; siete copas; 8 a 10 copas; 11 a 15 copas; más de 15 copas; no sabe. c) ¿Su pareja está de acuerdo con que usted trabaje fuera del hogar?: sí; no; no sé. d) ¿Quién de los dos, usted o su pareja, gana más dinero por su trabajo?: yo; igual remuneración; él; no sabe. Las diferentes categorías de las variables se ponderaron de acuerdo con la magnitud de su asociación con la violencia, y posteriormente se sumaron para obtener un indicador. Se establecieron tres categorías: a) bajo, b) mediano y c) alto.

La variable de estado emocional de la pareja en el hogar, se construyó a partir de la siguiente pregunta: ¿Cómo se siente la pareja cuando llega del trabajo? Las opciones de respuesta fueron: cansado; tenso; fastidiado; triste; alegre. Para su análisis se categorizaron en: no tensión (alegre); mediana (triste y cansado) y alta (tenso y fastidiado).

La variable de tensión laboral en la mujer, se generó a partir de las siguientes preguntas y opciones de respuestas: a) la relación con mis compañeros de trabajo es: constructiva; muy constructiva; indiferente; destructiva. b) El reconocimiento a mi trabajo que recibo del Instituto es: satisfactorio; muy satisfactorio; regular; insatisfactorio; muy insatisfactorio. c) Mis actividades cotidianas son: estimulantes; muy estimulantes; intermedias; rutinarias; muy rutinarias. d) Si pusiera en una balanza todo lo bueno y todo lo malo de su vivencia como trabajador del Instituto, el resultado sería: favorable; muy favorable; indeciso; desfavorable; muy desfavorable. Las diferentes categorías de las variables se ponderaron de acuerdo con la magnitud de su asociación con la violencia, y posteriormente se sumaron para obtener un valor continuo. Se establecieron, para su análisis, las siguientes categorías: no tensión, mediana y alta.

Por otra parte, se generó un indicador de antecedentes de violencia sufrida en la niñez de la mujer, así como aquel que existió entre sus padres. La combinación de estos reactivos permitió generar una variable con tres categorías para análisis: a) sin antecedentes de violencia; 
b) bajo, que corresponde a antecedentes por debajo de la mediana; c) alto, que son antecedentes por arriba de la mediana.

La categoría laboral de la mujer o actividad de trabajo se clasificó en tres categorías: a) médica; b) enfermeras, personal administrativo, trabajadoras sociales, nutriólogas, personal de salud; y c) intendencia.

\section{Análisis estadístice}

Se exploró la distribución de las variables de estudio entre los distintos tipos y grados de violencia de pareja. De acuerdo con distintas características sociodemográficas, se calculó la ocurrencia y la severidad de los diferentes tipos de violencia de pareja en el último año. Mediante análisis de regresión logística politómica ${ }^{23}$ se evaluó la asociación entre cada una de las variables de interés y las diferentes categorías del indicador de severidad de violencia de pareja. Se estimaron razones de momios (RM) crudas y ajustadas para cada variable de estudio y sus intervalos de confianza a 95\% (IC 95\%). Se utilizó el programa Stata versión 8 (Stata corp., Texas, EUA) para realizar el análisis estadístico de la información.

\section{Resultados}

\section{Garacterísticas de la población}

La edad promedio de la población analizada ( $\mathrm{n}=1$ 173) fue de 40.8 años $(D E \pm 7.9)$, con un rango de edad de 21 a 77 años; alrededor de $45 \%$ se ubicó en el grupo de edad más joven ( $<40$ años). La edad promedio de

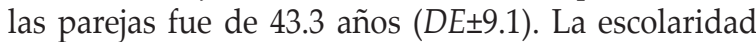
promedio de las mujeres, así como de sus parejas, fue de 13.3 años. Cerca de $50 \%$ de las mujeres y $44 \%$ de sus parejas realizaron estudios superiores. De las mujeres, $80.9 \%$ informaron estar casadas y el resto indicó que vivía en unión libre.

\section{Prevalencia de violencia de pareja}

La prevalencia de cualquier tipo de violencia de pareja fue de 42.3 por ciento. Por tipos de violencia, se encontró que la prevalencia de violencia psicológica fue la más alta $(37.7 \%)$, seguida de la violencia física $(23.4 \%)$ y la violencia sexual (9.5\%) (cuadro I).

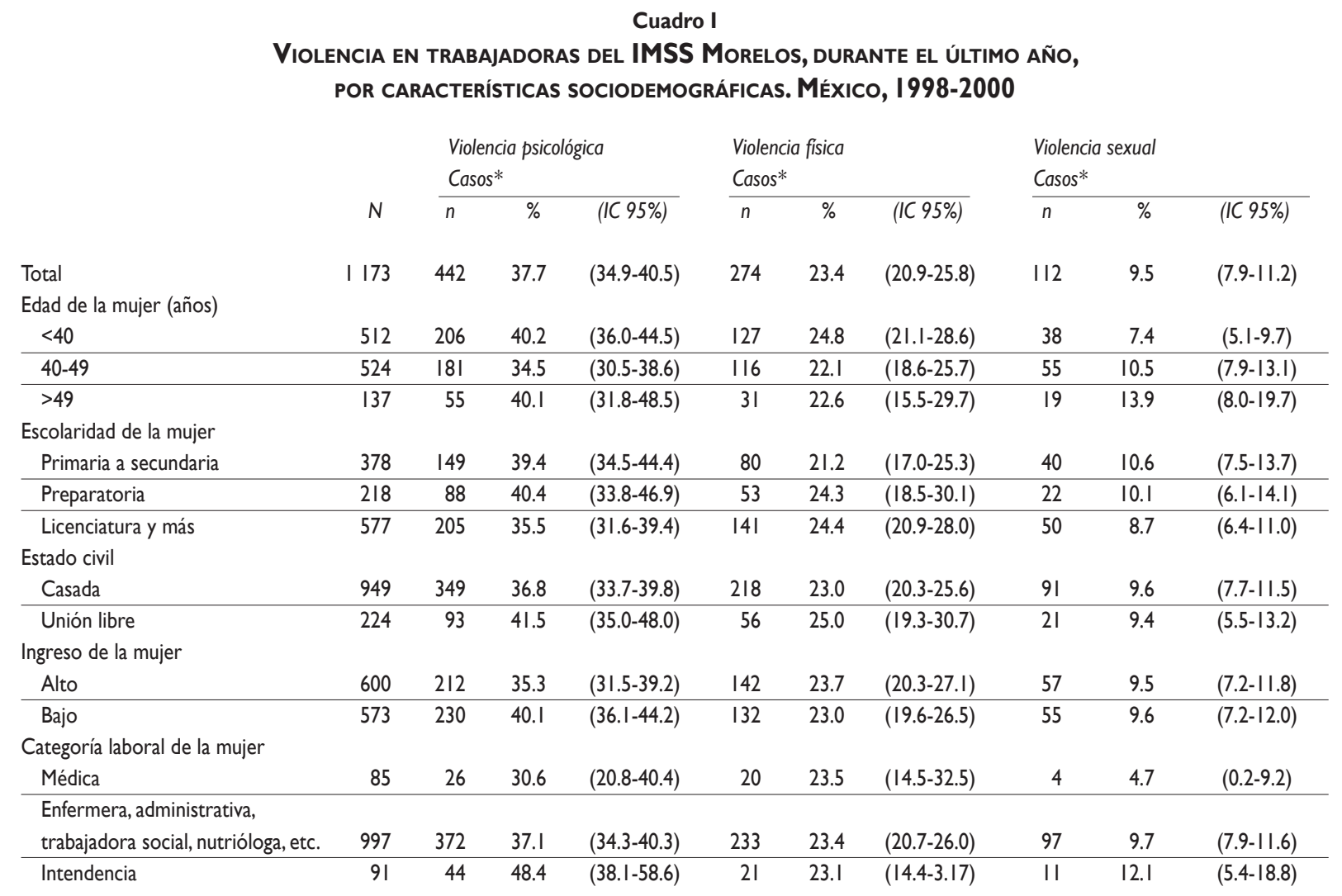

* Número y proporción de mujeres que refirieron haber sufrido algún tipo de violencia 
De acuerdo con la ponderación de la frecuencia relativa, las mujeres que notificaron niveles altos en los tres tipos de violencia fueron aquellas cuya pareja y ellas mismas refirieron tener menor escolaridad y vivir en unión libre, además de ser las mujeres con mayores antecedentes de violencia en la infancia. Aquellas mujeres mayores de 49 años, pertenecientes a la categoría de menor remuneración, informaron haber experimentado niveles más altos de violencia, tanto psicológica como sexual, siendo este grupo diferente al resto de la muestra y estadísticamente significativo (cuadro II).

\section{Severidad de la violencia}

Las mujeres que experimentan altos niveles de tensión en la relación de pareja, presentaron mayor riesgo de violencia de pareja durante el último año en comparación con aquellas que viven sin tensión en la relación, con un nivel de severidad moderado ( $R M=2.0$; IC 95\% $1.39-2.95)$ y para el severo ( $R M=6.7$; IC $95 \% 3.57-12.68)$. Las mujeres con una tensión laboral alta presentan mayor riesgo de violencia en ambos niveles de severidad: moderado ( $R M=1.7$; IC 95\% 1.25-2.36) y severo ( $R M=1.8$;

Cuadro II

Niveles de violencia en trabajadoras del IMSS, Morelos, POR Características demográficas. México, I998-2000

\begin{tabular}{|c|c|c|c|c|c|c|c|c|c|c|c|c|c|}
\hline & \multicolumn{7}{|c|}{$N=1 / 73$} & \multirow[b]{3}{*}{$p^{*}$} & \multirow{2}{*}{\multicolumn{3}{|c|}{ Sexual }} & \multirow[b]{3}{*}{$p^{*}$} \\
\hline & & & Psicológica & & \multirow[b]{2}{*}{$p^{*}$} & \multicolumn{3}{|c|}{ Física } & & & & & \\
\hline & & $\begin{array}{c}\text { No } \\
n=731 \\
\%\end{array}$ & $\begin{array}{c}\text { Baja-moderada } \\
n=286 \\
\%\end{array}$ & $\begin{array}{c}\text { Alta } \\
n=156 \\
\%\end{array}$ & & $\begin{array}{c}\text { No } \\
n=899 \\
\%\end{array}$ & $\begin{array}{c}\text { Baja-moderada } \\
n=193 \\
\%\end{array}$ & $\begin{array}{c}\text { Alta } \\
n=8 I \\
\%\end{array}$ & & $\begin{array}{c}\text { No } \\
n=1061 \\
\%\end{array}$ & $\begin{array}{c}\text { Baja } \\
n=43 \\
\%\end{array}$ & $\begin{array}{c}\text { Alta } \\
n=69 \\
\%\end{array}$ & \\
\hline \multicolumn{14}{|l|}{ Escolaridad de la mujer } \\
\hline Primaria a secundaria & 378 & 60.6 & 22.5 & 16.9 & & 78.8 & 14.0 & 7.1 & & 89.4 & 2.4 & 8.2 & \\
\hline Preparatoria & 218 & 59.6 & 26.6 & 13.8 & & 75.7 & 16.5 & 7.8 & & 89.9 & 3.7 & 6.4 & \\
\hline Licenciatura y más & 577 & 64.5 & 24.8 & 10.7 & $<0.001$ & 75.6 & 18.0 & 6.4 & 0.714 & 91.3 & 4.5 & 4.2 & $<0.001$ \\
\hline \multicolumn{14}{|l|}{ Escolaridad de la pareja } \\
\hline Primaria a secundaria & 328 & 55.8 & 25.9 & 18.3 & & 72.9 & 18.6 & 8.5 & & 87.2 & 3.7 & 9.1 & \\
\hline Preparatoria & 230 & 62.6 & 27.0 & 10.4 & & 74.8 & 19.1 & 6.1 & & 88.3 & 6.1 & 5.7 & \\
\hline Licenciatura y más & 513 & 66.9 & 21.8 & 11.3 & $<0.001$ & 80.5 & 13.8 & 5.7 & 0.006 & 93.4 & 2.5 & 4.1 & $<0.001$ \\
\hline \multicolumn{14}{|l|}{ Edad de la mujer (años) } \\
\hline$<40$ & 512 & 59.8 & 28.5 & 11.7 & & 75.2 & 17.4 & 7.4 & & 92.6 & 2.5 & 4.9 & \\
\hline $40-49$ & 524 & 65.5 & 20.6 & 13.9 & & 77.9 & 15.1 & 7.1 & & 89.5 & 4.6 & 5.9 & \\
\hline$>49$ & 137 & 59.9 & 23.4 & 16.8 & 0.0 & 77.4 & 18.2 & 4.4 & 0.066 & 86.1 & 4.4 & 9.5 & 0.002 \\
\hline \multicolumn{14}{|l|}{ Estado civil } \\
\hline Casada & 949 & 63.2 & 24.6 & 12.2 & & 77.0 & 16.9 & 6.1 & & 90.4 & 4.0 & 5.6 & \\
\hline Unión libre & 224 & 58.5 & 23.7 & 17.9 & $<0.001$ & 75.0 & 14.7 & 10.3 & 0.002 & 90.6 & 2.2 & 7.1 & 0.227 \\
\hline \multicolumn{14}{|l|}{ Categoría laboral de la mujer } \\
\hline Médica & 686 & 69.4 & 21.2 & 9.4 & & 76.5 & 17.7 & 5.9 & & 95.3 & 1.2 & 3.5 & \\
\hline \multicolumn{14}{|l|}{ Enfermera, administrativa, } \\
\hline Intendencia & 91 & 51.7 & 29.7 & 18.7 & 0.001 & 76.9 & 18.7 & 4.4 & 0.529 & 87.9 & 3.3 & 8.8 & 0.030 \\
\hline
\end{tabular}

Antecedentes de violencia

en la mujer

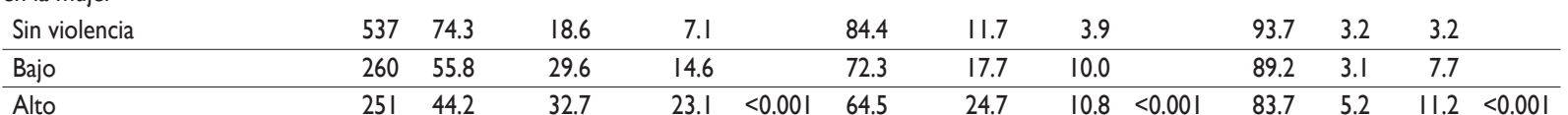

* Valor p para diferencia de proporciones entre categorías de exposición 
IC 95\% 1.14-2.76). Aquellas mujeres cuyas parejas presentan altos niveles de tensión laboral tienen un riesgo tres veces mayor en el nivel de severidad moderado ( $R M=3.0$; IC 95\% 1.76-5.08) y 10.9 veces mayor riesgo de sufrir agresiones por parte de su pareja $(R M=10.9$; IC 95\% 5.54-21.71) (cuadro III).

En el nivel severo del indicador de severidad de violencia de pareja, las mujeres con un historial importante de violencia en la niñez presentan un riesgo 2.3 veces mayor de sufrir violencia de pareja $(R M=2.3$; IC $95 \%$ 1.63-3.38) y casi cinco veces más posibilidades de haber sufrido violencia en el último año $(R M=4.5 \mathrm{IC}$ 95\% 2.81-7.35) (cuadro III).

\section{Discusión}

El presente estudio indica una alta prevalencia de violencia de pareja en la muestra de trabajadoras de la salud en el IMSS Morelos. Los principales hallazgos del análisis sugirieron que ser joven, vivir en unión libre y pertenecer a las categorías laborales menos remuneradas, constituyen elementos de riesgo para que ocurra la

Cuadro III

FACTORES ASOCIADOS A DISTINTAS CATEGorías DEL ÍNDICE DE SEVERIDAD DE VIOLENCIA durante el Último año entre mujeres trabajadoras del IMSS, Morelos. México, 1998-2000

\begin{tabular}{|c|c|c|c|c|c|c|c|}
\hline & \multirow{2}{*}{$\begin{array}{l}\text { Sin } \\
\text { violencia } \\
n=677 \\
\%\end{array}$} & \multicolumn{3}{|c|}{$\begin{array}{c}\text { Indicador de severidad de violencia } \\
\text { Bajo-Moderado }\end{array}$} & \multicolumn{3}{|c|}{ Alto } \\
\hline & & $n=333$ & $\begin{array}{c}R M \\
\%\end{array}$ & (IC $95 \%)$ & $\begin{array}{c}n=163 \\
\%\end{array}$ & $R M$ & (IC 95\%) \\
\hline \multicolumn{8}{|l|}{ Edad de la mujer (años) } \\
\hline$<40$ & 47.7 & 14.3 & 1.64 & $1.22-2.20$ & 48.5 & 0.88 & $0.58-1.34$ \\
\hline $40-49$ & 41.2 & 12.4 & 1.00 & & 36.8 & 1.00 & \\
\hline$>49$ & II.I & 3.3 & 1.37 & $0.85-2.23$ & 14.7 & 1.25 & $0.68-2.31$ \\
\hline Prueba de tendencia & & & 0.22 & & & 0.06 & \\
\hline
\end{tabular}

Indicador de relación de pareja y consumo de alcohol

\begin{tabular}{|c|c|c|c|c|c|c|c|}
\hline Bajo & 27.6 & I7.4 & 1.00 & & 8.0 & 1.00 & \\
\hline Mediano & 32.8 & 29.4 & 1.33 & $0.89-1.98$ & 13.5 & 1.39 & $0.66-2.93$ \\
\hline Alto & 32.5 & 46.5 & 2.03 & I.39-2.95 & 72.4 & 6.73 & $3.57-12.68$ \\
\hline
\end{tabular}

Estado emocional de la pareja en el hogar

\begin{tabular}{lrrrrrrrr} 
No tensión & 32.3 & 17.7 & 1.00 & & 10.4 & 1.00 & \\
\hline Mediana & 6.9 & 59.2 & 2.06 & $1.45-2.93$ & 29.4 & $2.7 \mid$ & $1.52-4.84$ & 1 \\
\hline Alta & 48.9 & 12.3 & 2.99 & $1.76-5.08$ & 47.9 & 10.97 & $5.54-21.71$ &
\end{tabular}

Tensión laboral de la mujer

\begin{tabular}{|c|c|c|c|c|c|c|c|}
\hline No tensión & 41.8 & 33.9 & 1.00 & & 32.5 & 1.00 & \\
\hline Mediana & 21.1 & 18.0 & 1.13 & $0.76-1.66$ & 17.2 & 1.15 & $0.66-2.00$ \\
\hline Alta & 31.6 & 44.4 & 1.72 & $1.25-2.36$ & 42.9 & 1.78 & I.I4-2.76 \\
\hline Prueba de tendencia & & & 0.001 & & & 0.30 & \\
\hline
\end{tabular}

Antecedentes de violencia en la mujer

\begin{tabular}{|c|c|c|c|c|c|c|c|}
\hline Sin violencia & 54.7 & 37.2 & 1.00 & & 26.4 & 1.00 & \\
\hline Bajo & 20.2 & 25.5 & 1.75 & $1.23-2.49$ & 23.3 & 2.34 & $1.40-3.92$ \\
\hline Alto & 14.9 & 25.8 & 2.34 & $1.63-3.38$ & 39.3 & 4.54 & $2.81-7.35$ \\
\hline Prueba de tendencia & & & 0.001 & & & $<0.001$ & \\
\hline \multicolumn{8}{|l|}{ Categoría laboral de la mujer } \\
\hline Médica & 8.4 & 5.4 & 1.00 & & 6.1 & 1.00 & \\
\hline \multicolumn{8}{|l|}{ Enfermera, administrativa, } \\
\hline trabajadora social, nutrióloga, etc. & 85.7 & 83.8 & 1.53 & $0.86-2.71$ & 84.7 & 1.54 & $0.70-3.38$ \\
\hline Intendencia & 5.9 & 10.8 & 2.58 & I.24-5.34 & 9.2 & 1.81 & $0.66-4.95$ \\
\hline
\end{tabular}

El modelo fue ajustado por edad de la mujer; tensión en la relación de pareja y consumo de alcohol; estado emocional de la pareja en el hogar; tensión laboral de la mujer; antecedentes de violencia en la mujer y categoría laboral de la mujer 
violencia de pareja. Adicionalmente, los datos sugieren que la tensión experimentada en el trabajo, emerge como un importante determinante de riesgo de violencia de pareja. De igual manera, el antecedente de violencia en la infancia es un factor de riesgo mayor para experimentar la violencia en la edad adulta, como se ha descrito en investigaciones previas. ${ }^{5,13,17,24-27}$

Las prevalencias de violencia observadas en este estudio son semejantes a las notificadas en investigaciones previas realizadas en el estado de Morelos. ${ }^{17} \mathrm{Se}$ observa que predomina la violencia psicológica sobre la violencia física y la sexual, tal cual se ha reportado en investigaciones hechas en los planos nacional e internacional; así, se han notificado prevalencias de cerca de $30 \%$ en estudios hechos en Estados Unidos y Europa, y de 50 a $60 \%$ en investigaciones realizadas en países latinoamericanos. ${ }^{5,8,10,13,28-32}$ Sin embargo, los datos del presente trabajo muestran una prevalencia dos veces más alta que la informada en la Encuesta Nacional de Violencia contra las Mujeres (ENVIM-2003), para todos los tipos de violencia de pareja, y que fuera de $19.6 \%$ (psicológica); $9.8 \%$ (física), y 7\% (sexual), de tal manera que en la presente muestra se encontraron prevalencias de 37.7, 23.4 y 9.5\%, respectivamente. Una razón de esto podría ser la diferencia en los instrumentos utilizados, la medición y la conceptualización de los diferentes tipos de violencia en ambos estudios.

Tanto en el presente estudio como aquellos realizados previamente en el estado de Morelos, para cada tipo de la violencia de pareja se utilizaron preguntas adicionales relacionadas con los eventos ocurridos con mayor frecuencia y que posiblemente llevan a altas prevalencias de violencia observadas en estos estudios. Otra razón podría residir en el hecho de que las participantes en esta investigación contestaron cuestionarios de auto aplicación en la intimidad de sus hogares u oficinas (a diferencia de las entrevistas y cuestionarios aplicados en otros estudios). Los cuestionarios de autoaplicación son más apropiados para obtener respuesta a las preguntas íntimas tales como aquellas relacionadas con la violencia de pareja; al responderlas en un ámbito privado, las mujeres generalmente se sienten menos inhibidas. Por otra parte, esta muestra está constituida, en gran parte, por mujeres de clase media o de clase media baja que trabajan en el sector salud con, al menos, un nivel de educación media superior. Así, es probable que se sientan menos inhibidas y que les parezca menos difícil entender el cuestionario y contestar las preguntas de manera honesta, en comparación con lo que sucede con la muestra representativa nacional, dirigida a mujeres más pobres y que no trabajan.

Por otra parte, se encontró que las mujeres jóvenes tienen un mayor riesgo de vivir la violencia de pareja en comparación con las mujeres de mayor edad. A pesar de que la posición socioeconómica no está relacionada con la violencia de pareja en el análisis ajustado, en varios estudios se ha observado que el fenómeno de violencia doméstica rebasa la clase social. Las altas prevalencias de violencia doméstica se observan tanto en mujeres ricas como en pobres; entre estas últimas se observaron prevalencias de violencia de pareja levemente mayores. ${ }^{5,17,26}$ La ausencia de asociación observada en la muestra del presente estudio podría deberse a la poca variabilidad en el nivel socioeconómico (no hubo mujeres pertenecientes a los espectros sociales muy alto y muy bajo). Sin embargo, existe una diferencia en las mujeres de la clase alta y aquellas de clase baja en términos de su acceso a recursos para combatir la violencia de pareja, y de ahí su impacto en el bienestar psicosocial de las mismas. Estas son las preguntas teóricas que ameritan mayor investigación en el futuro.

En este trabajo se encontró que las mujeres con mayores diferencias en su relación de pareja presentaron más posibilidades de ser víctimas de violencia tanto moderada como severa; estos hallazgos coinciden con los de otros estudios. ${ }^{18-20,26}$ La tensión en el trabajo, tanto para hombres como para mujeres, está relacionada con altos niveles de violencia de pareja; en la medida en que aumenta el nivel de tensión laboral, también se eleva el riesgo para las mujeres de ser víctimas de la violencia, así como la severidad de la misma. La incorporación formal de la mujer al mercado laboral ha dado lugar a cambios; sin embargo, a pesar de que se ha flexibilizado la división social del trabajo, la responsabilidad de los quehaceres domésticos sigue recayendo sobre todo en las mujeres, independientemente de su participación en el mercado laboral. ${ }^{33}$ Debido a que la carga de trabajo familiar de las mujeres no ha cambiado, el incremento de responsabilidades aumenta las dificultades de comunicación, elevando el nivel de tensión, favoreciendo que se perpetúe el ciclo de violencia de pareja.

Adicionalmente, las mujeres con altos niveles de antecedentes de violencia presentaron casi cinco veces más riesgo de padecer violencia de pareja ( $R M=4.5$ IC $95 \%$ 2.81-7.35). Estos resultados son consistentes con los de otras investigaciones, lo cual sugiere que las conductas violentas podrían ser aprendidas de las experiencias de la infancia; el hecho de ser testigo de violencia intrafamiliar es un predictor de riesgo de violencia en edades adultas. ${ }^{5,13,17,24-27}$ Varios investigadores han demostrado que un número importante de delincuentes han vivido en el seno de familias violentas, y que la violencia familiar se reproduce en las familias, de generación en generación. ${ }^{28,34}$

Las tensiones parecen ser los elementos disparadores de los episodios violentos que van más allá del 
control de hombres y mujeres. Esto sugiere que una intervención en el manejo de los problemas podría resultar en una disminución de la violencia doméstica. La exposición a la violencia en la infancia es otro factor del riesgo asociado con la violencia de pareja, lo cual sugiere que la violencia es una conducta aprendida que impacta a las generaciones. Las normas culturales dentro de la familia enseñan a los niños la tolerancia hacia la violencia como una manera legítima de liberar la tensión o de controlar a los demás, especialmente en el caso de los hombres. La implicación de este hallazgo es que enseñar a los niños a romper con esas normas culturales y a aprender otras formas de manejar mejor los problemas, pudiera ayudar a reducir la violencia intrafamiliar a través del tiempo.

Los estudios de violencia de pareja han carecido de instrumentos que puedan ser usados para comparar y estudiar los factores de riesgo asociados. La mayoría de los instrumentos han tomado en cuenta sólo las prevalencias para estudiar la violencia de pareja. Sin embargo, cada tipo de violencia difiere en su impacto sobre el bienestar físico y psicológico de las mujeres. Tratar cada acontecimiento de igual manera, podría no representar la situación real de la severidad de la violencia doméstica y su impacto en el bienestar psicosocial de las mujeres. En este estudio se constituyó un indicador de severidad de violencia que mide la percepción de severidad de las mujeres para cada evento, ponderando cada acto de violencia. Por lo tanto, este indicador muestra cómo los eventos más frecuentes son más severos, al compararlos con los menos severos. Roberto Castro y colaboradores ${ }^{5}$ utilizaron este enfoque previamente, con excelentes resultados. Además, se encontró que este enfoque mejora los resultados al incrementar la magnitud de las asociaciones.

A partir de las altas tasas de violencia de pareja que se observan en esta investigación, así como aquellas encontradas en estudios previos, es evidente la necesidad de impartir conocimiento y capacitación al personal de salud..$^{22}$ Asimismo, es indispensable la creación de programas de prevención, dirigidos a este sector laboral, que generen un cambio en los patrones culturales que legitiman la violencia, además de que provean de apoyos especializados a las víctimas de la misma, sin dejar de lado la atención a los victimarios, incluyéndolos en programas para manejar la tensión, por ejemplo.

Entre las limitaciones de los estudios transversales se encuentra su ambigüedad temporal y la no obtención de datos de la pareja en forma directa. Debido a las características de la población de estudio, los resultados de esta investigación podrán extrapolarse a poblaciones de mujeres trabajadoras con características similares.

\section{Referencias}

I.World Health Organization. WHO/WHD Violence against women: a priority health. Geneva:World Health Organization, 1997.WHO document WHO/FRH/WHD/97.8\#.

2. Uribe-Elías R, Billings D.Violencia sobre la salud de las mujeres ¿Por qué hoy? Femengo/lpas México, 2003.

3. Taket A, Nurse J, Smith K, Watson J, Hakespeare J, Lavis V et al. Routinely asking women about domestic violence in health settings. BMJ 2002:327:673-676.

4. Rohlfs I,Valls-Llobet. Taking action against domestic violence. A challege for public health. Gac Sanit 2003; 17:263-265.

5. Castro R, Peek-Asa C, Ruiz A.Violence against women in Mexico: a study of abuse before and during pregnancy.Am J Public Health 2003;93:1110-1116.

6. Golberg WG, Tomlanovich MC. Domestic violence victims in the emergency department. New findings. JAMA 1984;25I(24):3259-3264. 7. Candib LM.Violence against women: no more excuses. Fam Med 1989;2I(5):339-342.

8.Valdez-Santiago R, Sanín-Aguirre LH. Violencia doméstica durante el embarazo y su relación con el peso al nacer. Salud Publica Mex 1996;38:352-362

9. Soreson SB, Upchurch DM, Shen H.Violence and injury in marital arguments: risk patterns and gender differences. Am J Public Health 1996;86:35-40.

10.Wijma B, Schei B, Swahnberg K, Hilden M, Offerdal K, Pikarinen U et al. Emotional, physical, and sexual abuse in patients visiting gynaecology clinics: a Nordic cross-sectional study. Lancet 2003;36I:2 I07-2 I I3. II. Morrison A, Ellsberg M, Bott S. Addressing gender-based violence in the Latin American and Caribbean region: a critical review of interventions. World Bank Policy Working Paper 3438 and PATH, October 2004. Washington, DC.

12. Norton LB, Peipert JF, Zierler S, Lima B, Hume L. Battering in pregnancy: an assessment of two screening methods. Obstet Gynecol 1995;85:32|-324

13. Olaiz G, Rico B, Del Río A. Encuesta Nacional sobre Violencia contra las Mujeres. Secretaría de Salud, INSP, 2004.

14. Encuesta Nacional de Salud Reproductiva con población derechohabiente. ENSARE I998. IMSS, 2000.

I5. Encuesta Nacional sobre Dinámica de las Relaciones en los Hogares. ENDIREH 2003. INEGI. 2004.

16.-Ramírez-Rodríguez JC, Uribe-Vázquez G. Mujer y violencia: un hecho cotidiano. Salud Publica Mex 1993;35: 148-160.

I7. Rivera-Rivera L, Lazcano-Ponce E, Salmerón-Castro J, SalazarMartínez E, Castro R, Hernández-Avila M. Prevalence and determinants of male partner violence against Mexican women. Salud Publica Mex 2004;46: I I3-1 22.

18. O'Campo P, Gielen A, Faden R.Violence by male partners against women during the childbearing year: a contextual analysis. Am J Public Health 1995;85:1092-1097.

19. Koenig MA, Lutalo T, Zhao F,Wabwire-Mangen F, Kiwanuka N,Wagman J et al. Domestic violence in rural Uganda: evidence from a communitybased study. Bull World Health Organ 2003;8I:53-60.

20. Biernat M,Wortman C. Sharing of home responsabilities between professional employed women and their husbands. J Pers Soc Psychol 1991;60(6):844-860.

21. Natera GR, Tiburcio MS, Villatoro JV. Marital violence and its relationship to excessive drinking in Mexico. Contemp Drug Probl 1997;24(4):787-804.

22. Mendez-Hernández P,Valdez-Santiago R, Viniegra-Velázquez L, RiveraRivera L, Salmerón Castro J.Violencia contra la mujer. Conocimiento y 
actitud del personal médico del Instituto Mexicano del Seguro Social, Morelos, México. Salud Publica Mex 2003;45:472-482.

23. Hosmer DW, Lemeshow S. Applied logistic regresión. Wiley-

Interscience Publication. Special Topics: polytomous logistic regression. United States of America 1989:216-245.

24.Alvarado-Zaldivar G, Slavador-Moysén J, Estrada-Martínez S, TerronesGonzález A. Prevalencia de violencia doméstica en la ciudad de Durango. Salud Publica Mex 1998;40:48I-486.

25. Natera-Rey G, Juárez-García F,Tiburcio-Sainz M. Validez factorial de una escala de violencia hacia la pareja en una muestra nacional mexicana. Salud mental 2004;27:3I-38.

26. Ellsberg MC, Peña R, Herrera A, Lijestrand J,Winkvist A. Wife abuse among women of childbearing age in Nicaragua. Am J Public Health 1999;89(2):241-244.

27. Tuesca R, Borda M. Violencia física marital en Barranquilla (Colombia): prevalencia y factores de riesgo. Gac Sanit 2003;4:302-308.

28. Krug E, Dahlberg L, Mercy J, Zwi A, Lozano R, eds. Informe mundial sobre la violencia y la salud. Organización Mundial de la Salud. Ginebra: OMS-OPS, 2002.
29. Duarte P. Encuesta de opinión pública sobre la incidencia de violencia en la familia.Asociación Mexicana contra la Violencia hacia las Mujeres AC (COVAC). México, DF: Fondo de Población de las Naciones Unidas/ Procuraduría General de Justicia del Distrito Federal, 1995:17.

30. Ramírez RJC, Patiño G. Mujeres de Guadalajara y violencia doméstica: resultados de un estudio piloto. Cad Saude Publica 1996; 12:405-409.

3I. Schei B, Bakketeig LS. Gynaecological impact of sexual and physical abuse by spouse. A study of a random sample of Norwegian women. $\mathrm{Br}$ J Obstet gynecol I8989;96(I2):1379-1383.

32. Jewkes R, Penn-Kekana L,Levin J, Ratsaka M, Schrieber M. Prevalence of emotional, physical and sexual abuse of women in three South African provinces. S Afr Med J 2001;9I(5):42I-428.

33. Ministerio de Asuntos Sociales. Mujeres latinoamericanas en cifras. Bogotá: Flasco, 1995.

34. Martin SL, Moracco KE, Garro J, Ong Tsui A, Kupper LL, Chase JL et al. Domestic violence across generations: findings from northern India. Int J Epidemiol 2002;31:560-572 
Anexo I

LAS SIGUIENTES PREGUNTAS BUSCAN INDAGAR SOBRE LA VIOLENCIA QUE HAN SUFRIDO LAS MUJERES, ASÍ COMO SU PERCEPCIÓN DE LA SEVERIDAD DE LAS DIFERENTES FORMAS DE VIOLENCIA

\begin{tabular}{|c|c|c|c|c|c|c|}
\hline Durante los últimos 12 meses, su pareja: & Nunca & Una vez & Varias veces & Muchas veces & Alguna vez en la vida & Valor de I a 100 \\
\hline \multicolumn{7}{|l|}{ I) ¿La ha empujado a propósito? } \\
\hline \multicolumn{7}{|l|}{ 2) ¿La ha sacudido, zarandeado o jaloneado? } \\
\hline \multicolumn{7}{|l|}{ 3) ¿Le ha torcido el brazo? } \\
\hline \multicolumn{7}{|l|}{ 4) ¿La ha jaloneado del pelo? } \\
\hline \multicolumn{7}{|l|}{ 5) ¿La ha golpeado con la mano o el puño? } \\
\hline \multicolumn{7}{|l|}{ 6) ¿La ha pateado? } \\
\hline \multicolumn{7}{|l|}{ 7) ¿Le ha golpeado el estómago? } \\
\hline \multicolumn{7}{|l|}{ 8) ¿Le ha aventado algún objeto? } \\
\hline \multicolumn{7}{|l|}{ 9) ¿La ha encerrado bajo llave? } \\
\hline \multicolumn{7}{|l|}{ 10) iLa ha amarrado? } \\
\hline \multicolumn{7}{|l|}{$\begin{array}{l}\text { II) ¿La ha golpeado con algún palo, } \\
\text { cinturón o algún objeto doméstico? }\end{array}$} \\
\hline \multicolumn{7}{|l|}{ 12) ¿Le ha quemado con un cigarro? } \\
\hline \multicolumn{7}{|l|}{ 13) ¿La ha tratado de ahorcar o asfixiar? } \\
\hline \multicolumn{7}{|l|}{ 14) ¿La ha agredido con alguna navaja, cuchillo o machete? } \\
\hline \multicolumn{7}{|l|}{ 15) ¿Le ha disparado con una pistola o rifle? } \\
\hline \multicolumn{7}{|l|}{ 16) ¿La rebaja o menosprecia? } \\
\hline \multicolumn{7}{|l|}{ 17) ¿La insulta? } \\
\hline \multicolumn{7}{|l|}{ 18) ¿Se puso celoso o sospecha de sus amistades? } \\
\hline \multicolumn{7}{|l|}{ 19) ¿Le dice cosas como que está poco atractiva o fea? } \\
\hline \multicolumn{7}{|l|}{ 20) ¿Se puso a golpear o a patear la pared o algún mueble? } \\
\hline \multicolumn{7}{|l|}{ 2I) ¿Se puso a destruir algunas de sus cosas? } \\
\hline \multicolumn{7}{|l|}{ 22) ¿La amenazó con golpearla? } \\
\hline \multicolumn{7}{|l|}{ 23) ¿La amenazó con alguna navaja, cuchillo o machete? } \\
\hline \multicolumn{7}{|l|}{ 24) ¿La amenazó con alguna pistola o rifle? } \\
\hline \multicolumn{7}{|l|}{ 25) ¿Le hizo sentir miedo de él? } \\
\hline \multicolumn{7}{|l|}{ 26) ¿La amenazó con matarla? } \\
\hline \multicolumn{7}{|l|}{ 27) ¿La amenazó con matarse a sí mismo? } \\
\hline \multicolumn{7}{|l|}{ 28) ¿Se opone a que trabaje o estudie? } \\
\hline 29) ¿Siente usted que la controla o vigila? & & & & & & \\
\hline 30) ¿Le impide que visite a sus amistades o familiares? & & & & & & \\
\hline $\begin{array}{l}\text { 31) ¿Le ha exigido tener relaciones sexuales } \\
\text { aunque usted no esté dispuesta? }\end{array}$ & & & & & & \\
\hline $\begin{array}{l}\text { 32) ¿La amenaza con irse con otras mujeres } \\
\text { si no accede a tener relaciones sexuales con él? }\end{array}$ & & & & & & \\
\hline $\begin{array}{l}\text { 33) ¿Usa la fuerza física para tener relaciones } \\
\text { sexuales con usted contra su voluntad? }\end{array}$ & & & & & & \\
\hline 34) ¿La obliga a tener sexo oral en contra de su voluntad? & & & & & & \\
\hline 35) ¿La obliga a tener sexo anal en contra de su voluntad? & & & & & & \\
\hline
\end{tabular}

